\title{
LOS ORDINALES EN LA FAMILIA MAYANCE Y ALGUNAS LENGUAS TÚRQUICAS
}

\author{
Elcanor Fraske: \\ INAH, Departamento de Lingüística
}

Cada categoría morfológica y sintáctica expresa y refleja, en alguna forma especifica, el pensamiento de los hablantes. Por estudios conducidos por Ivar Aasen en el siglo xxx sobre el idioma noruego, Haugen aclara precisamente que "a través del estímulo de su percepción creciente de la gramática se le indujo dar énfasis a la morfología que consta de los criterios realmente más importantes de un idioma" (1965: 193). Siguiendo este punto de vista, podemos captar el cuadro auténtico del grupo mayance que nos ofrecen los ordinales.

Empezamos con la definición de Lázaro de un ordinal como "adjetivo o sustantivo numeral que expresa el lugar que algo ocupa en una serie o sucesión, primero, segundo, tercero, etc." (1971: 305), que es hasta cierto punto clara. Él omitió mencionar un aspecto morfológico de suma importancia, es decir, el hecho de que los ordinales nunca existen como raíces primarias, sino resultan de derivaciones denominales. $\mathrm{Y}$ así, al igual que el leitmotivo en la música permite oir las variaciones del tema principal, también se pueden apreciar los ordinales con sus tonos de variaciones.

Resulta interesante que en el grupo mayance los ordinales se distingan de otros grupos lingüísticos, por ejemplo del túrquico que tiene un conjunto de morfemas fijos para formar los ordinales. Estos elementos están sujetos a una modificación vocálica y consonántica según el ambiente fonológico que la determina de acuerdo al esquema fonológico de cada idioma. Por otro lado, el grupo mayance manifiesta muchos tipos de morfemas para producir los ordinales. Además, hay algunos idiomas en este grupo que carecen del concepto de ordinal. Me refiero al lacandón, al mopán y al maya 
itzá del Petén, aunque el yucateco que, con los tres idiomas citados, constituye lo que Romero designa "maya peninsular" (1977), sí conoce y utiliza este concepto a su manera. Lo que resulta en el grupo mayance varía del rango de un ordinal hasta una derivación que modifica el sentido general, precisando el número del día que corresponde a la raíz numérica.

Hay tres grupos morfémicos: sufijos, prefijos, y prefijos combinados con sufijos, que derivan los ordinales en el grupo mayance.

\section{I.1 - -aj / -ej / -ij}

Estas unidades exhiben un factor común: una sola consonante, la aspirada laríngea fortis / $\mathrm{j}$ / combinada con tres vocales en valores distintos. Mientras que se emplea $-a j$ en el quiché para derivar los ordinales que son sustantivos, las otras combinaciones pertenecen al cakchiquel, al chuj, al quiché, al tseltal y al tsotsil que no derivan ordinales en el sentido general, sino expresan un aspecto relacionado con un ordinal por decir "dentro de..." con referencia a numerales que son cardinales. Del sufijo $-e j$ en el chuj, Hopkins dice que "deriva sustantivos de numerales para describir una distancia temporal hacia el futuro" (1967: 98). Este concepto es, por un lado, más limitado que un ordinal y, por otro, más preciso en el aspecto temporal. Schumann designa $-i j$ en el quiché como un sufijo "para contar días en el futuro" (1976-77: 248).

lajujaj qui "diezmo" < lajuj "diez" (Edmonson, 1965: 65). belejej cakchi "dentro de nueve días", en otras palabras, en el no veno día < belej "nueve" (CE, 1940: 58).

ošij cakchi qui / ošej chuj "dentro de tres días", que es equivalente al tercer día / *šej ptsel tso "hoy en tres días" < oš "tres" (CE, 1940: 299 / Hopkins, 1967: 98 / Kaufman, 1972: 113). *čonej ptso "de hoy en cuatro días", que significa en el cuarto día (Kaufman, 1972: 99) < *čon < čan "cuatro".

Como se sabe "a veces y en algunos dialectos del tsotsil, la / - a- / de la raíz del tseltal se presenta como / - o- / ' (Frankle, 1980: 81). Para el tsotsil de San Andrés, Delgaty apunta que la / o / de la base nominal se desarrolla en / a /, por ejemplo, čon "víbora, animal" > sčanul "su animal" por agregar el sufijo -ul (1961: 418) sin mencionar que la vocal radical en el mayance general es / a /. En su trabajo más reciente Hurley 
(1978) no aclara tales diferencias. Es importante notar que la modificación en la raíz radical, como sucede en el tsotsil, no ocurre cuando se forman composiciones en las cuales la / o / $</$ a / mayance general constituye la última parte: ajačon "víbora de cascabel (Op. cit., 43).

ča'p'ej chuj "dentro de dos días" < ča'p' "dos" (Hopkins, 1967: 98).

ča'ej tso "pasado mañana", también es equivalente al segundo día $<$ ča' "dos" (Cowan, 1969: 42).

q'bij qui "pasado mañana < qab / qabi > q'b "dos" (Schumann, 1976-77: 248).

En los ejemplos citados y encontrados, solamente el quiché tiene dos tipos de morfemas fonológicos y morfológicos: $-a j$ para un ordinal sustantivo de un cardinal, mientras que $-i j$ tiene como función la de relacionar la derivación con el concepto temporal que refiere al numeral radical, y este sufijo coincide en su valor morfémico con el $-e j$ del cakchiquel, del chuj, del tseltal y del tsotsil.

\section{2 -či: 1}

El huasteco de San Luis Potosí es el único idioma mayance que emplea esta unidad morfémica; la utiliza en dos clases de derivaciones: ordinales y expresiones con el significado de "tal y tal vez" basado en el valor del numeral.

tsabči:l "segundo; la segunda vez" < tsa:b "dos" Larsen, 1955: 136, vii).

Las palabras para "dos", $t s a: b$ en el huasteco SLP, igual que las citadas anteriormente, revelan el desarrollo general de la oclusiva velar gutural tenuis $/ \mathrm{q} / 1$ del yucateco y del quiché a la africada alveopalatal / c c / en el chuj y en el tsotsil, y a la africada alveolar / ts / en el huasteco, lo que Swadesh había reconocido, designándolo "cambio" (1966: 177). Sin referirse a todos los idiomas mayances, el desarrollo específico caracteriza el grupo ge-

1 Aquí se distingue entre las oclusivas velar /q/ y palatal /k/ como se hace en la transcripción común para el grupo túrquico, un aspecto que se encuentra en la escritura misma del árabe, y que es también factible para la transcripción en el mayance. 
neral. Un aspecto interesante que sólo se encuentra en el huasteco de SLP es / ts / que corresponde al / č / en el huasteco de Veracruz: $\check{c} a: b$ que es típico del chuj y del tsotsil también.

ošči:l "tercer" < o:š "tres" (Larsen, 1955: 203). tse'či:l "la cuarta vez" < tse:' "cuatro" (Op. cit., vii, 80). buqči:l "séptimo" < bu:q "siete" (Op. cit., 190). aqaqči:1 "sexto; la sexta vez" < aqaq "seis" (Op. cit., 194, vii). lajujči:1 "décimo" < la:juj "diez" (Op. cit., 135).

Se puede uno dar cuenta de dos aspectos interesantes con base en los ejemplos citados arriba. Uno de éstos consta del hecho fonológico: cuando los morfemas contienen la vocal alargada, la vocal radical larga se reduce a una vocal sencilla. El otro, de índole diferente, surge de que el vocabulario en forma de diccionario implica que los significados en las dos secciones deben coincidir con las aclaraciones presentadas en la parte gramatical, mientras que la situación denuestra casos completamente diferentes.

\section{I.3. -itaqil}

Los morfemas -il abarcan muchas funciones en las derivaciones denominales. El cakchiquel presenta un caso distinto de los demás idiomas mayances en la formación de un ordinal, no a través de un cardinal, sino a través de una derivación en -itaq que expresa "veces" de los cardinales, a la cual se agrega el sufijo -il (Frankle, 1980: 104).

lajujitaqil "décimo" < lajujitaq "diez veces" < lajuj "diez" (CE, 1940: 244).

\section{I.4. - -lel}

En este caso se trata también de un idioma solamente, el chol, y hay que darse cuenta de que la unidad morfémica funciona no precisamente para derivar los ordinales, sino lo que Warkentin y Brend designan como "un marcador ordinal de clasificaciones numéricas" (1974: 91), es decir, "cuando se añade al número el sufijo -čajp para contar clases" (Frankle, 1980: 251).

čönčajplel "cuarto tipo" (Warkentin y Brend, 1974: 91) < čönčajp "cuatro clases" < cön < čan < qan yuca "cuatro". 
uščajplel "tercer tipo" (Aulie, 1978: 126) < uščajp "tres clases" $<$ uš "tres".

\section{I.5. $-\mathrm{nij}$}

También único al idioma chol, este sufijo deriva los ordinales directamente de los cardinales. Este tipo de ordinal refiere al tiempo preciso realcionado con la raíz numérica.

wašöknij "octavo día" (Aulie, 1978: 128) < *wašök < wašaq yuca "ocho".

wöknij "de hoy en seis días" (Op. cit., 133) es decir, en el sexto día $<$ *wök $<$ waq yuca "seis".

\section{I.6. -ňumel}

Esta unidad morfémica es de especial interés desde el punto de vista que caracteriza al chol general y al chol de Tila sin coincidir en el valor morfológico. Aulie dice que en el primer caso es "un sufijo numérico para contar vueltas, repetición" (1978: 98). A pesar de que no se le identifica con el concepto de un ordinal, de todos modos, tiene cabida aquí porque muestra la diferencia en la aplicación del mismo sufijo en un idioma y en uno de sus dialectos. El chol de Tila, según Schumann, emplea el sufijo para "los números oridnales, a excepción del primero" (1973: 31).

ča'ňumel chol T "segundo" < că' "dos" (Op. cit., 77, 30) cönňumel chol T "cuarto" / chol "cuatro veces o vueltas" (Schumann, Op. cit., 77/Aulie, 1978:52) < čön < qan yuca "cuatro". ušňumel chol T "tercero" / chol "tres veces" (Schumann, ibid. / Aulie, 1978:88) < uš < oš yuca "tres".

\section{I.7. -p'el}

El yucateco emplea los morfemas - $p$ 'el para derivar los ordinales de los cardinales.

lahunp'el "décimo" < lahun "diez" (SF, 1976: 209).

Cuando comparamos ésta con $(u)$ lahun yuca "décima parte; de 
diez uno; diezmo; décimo" (CORDEMEX, 1980: 432), que literalmente significa "su diez" se ve que hay más de una manera de derivar los ordinales. En este caso es a través del uso de $u$ el pronombre de la tercera persona que también expresa posesión. La formación citada expresa muchos significados; el de un ordinal es el último.

qa'p'el "partícula significa dos en cardinal u ordinal: dos, segun. do" (CORDEMEX, 301).

qa'p'el iši'm "dos granos de maíz" (ibid.)

\section{I.8. - tal}

Según Ochoa esta unidad "forma ordinales de los números en el huasteco de Veracruz" (1978: 134). Estos morfemas corresponden morfológicamente a la unidad $-\check{c} i: l$ del huasteco de SLP, sin tener las dos funciones de la última.

ča:btal "segundo" < ča:b "dos".

aqaqtal "sexto" < aqaq "seis".

hiø-tal "primero" (Ochoa, 1978: 20) $<$ *hiӨ.

Como en otros idiomas, la palabra para 'primero' "no tiene ninguna relación con la raíz para 'uno' en este idioma. En el huasteco SLP se encuentra yie 'primera vez, original' " (Frankle, 1980: 299 / Larsen, 1955: 92), presentando otro aspecto por la alternancia entre la semiconsonante / y / y la aspirada laríngea tenuis $/ \mathrm{h} /$.

\section{II.1. $\mathrm{r}-\mathrm{/} \mathrm{ru-}$}

Otra manera de formar los ordinales de los cardinales, que es característica de algunos idiomas mayances, consta del uso de prefijos correspondientes al pronombre posesivo de la tercera persona ante V y G respectivamente, cuyo significado es "su", "de él". Estos elementos sustantivizan al ordinal en el cakchiquel.

roš "el tercero" < oš "tres" (CE, 1940: 333, 297).

rubelej "el noveno" < belej "nueve" (Op. cit., 331, 58). 
II.2. $s-/ \mathrm{y} \cdot$

LaFarge y Bayers analizaron el uso de los morfemas citados que ocurren en el jacalteco. Aparte del ordinal para decir "primero", los demás ordinales "se forman regularmente por el prefijo pronominal de la tercera persona con los cardinales" (1931: 286). En este idioma los ordinales derivados de los cardinales funcionan solamente como atributos.

$$
\begin{aligned}
& \text { sqap "segundo" < qap "dos" } \\
& \text { yoš "tercero" < oš "tres" (ibid.) }
\end{aligned}
$$

III.1. s- / y- . - -al

El tercer grupo de derivaciones de ordinales consta de formaciones a través de una combinación de dos clases de morfemas: un prefijo con un sufijo. $\mathrm{Al}$ igual que en el jacalteco, los prefijos aparecen en el tsotsil en las mismas formas. $\mathrm{La}$ diferencia surge de la necesidad del sufijo -al que abarca muchas funciones en las derivaciones de sustantivos ya sean denominales, ya deverbales. Los ordinales que resultan de los cardinales a través de los morfemas combinados son sustantivos y adjetivos atributos. Jackobs y Longacre atribuyen cualidades a los morfemas -al que no son aplicables en estas derivaciones, ya que no coinciden con la función "del genitivo" (1967: 368), sino que son de categorías distintas. El posesivo es equivalente en estos casos al genitivo que se expresa por medio del prefijo; el pronombre de la tercera persona.

sčanibal "el cuarto" (Hurley, 1978: 460) < čanib "cuatro" (Op. cit., 37).

yošibal "el tercero" (Op. cit., 464) "tercero" (Jacobs y Longacre,

1967: 368) < ošib "tres" (Hurley, 1978: 95). svaqibal "el sexto" < vaqib "seis" (Op. cit., 214).

\section{III.1.a. s- / y- ... -il}

Los ordinales derivados de los cardinales a través del sufijo -il, en combinación con otro tipo de morfema, se encuentran en el chuj y en el yucateco con sus propias aplicaciones. Mientras que en el yucateco se agrega a un cardinal que lleva -tul, "el clasificador 
que refiere solamente a seres animados" (Swadesh, 1970: 83), el chuj requiere además "el prefijo del posesivo de la tercera persona: s- ante C, $y$ - ante V o / h /" (Frankle, 1980: 99). Hopkins aclara esto de otro modo al decir que el sufijo -il "también deriva bases poseídas de raíces numerales que parecen ser expresiones de ordinales y de ciclos" (1967: 95). Este análisis presenta algunas desviaciones de la realidad. El sufijo -il por sí mismo "no deriva en estos casos sustantivos poseídos, sino que es la combinación de este sufijo con el prefijo pronominal del posesivo de la tercera persona" (Frankle, 1980: 99). Además, referir a estas derivaciones como 'parecen ser expresiones de numerales...', tampoco es exacto cuando pertenecen a tal grupo categóricamente.

sča'p'il chuj "segundo" < ča'p' "dos"

y'ošil chuj "tercero" < 'oš "tres"

yhoil chuj "quinto" $<$ ho" "cinco"

slajunil chuj "décimo" < lajun "diez" (Hopkins, 1967: 95).

oštulil yuca "el tercero" (Arzápalo, 1973: 81).

Hay que darse cuenta de que esta derivación se utiliza solamente cuando se refiere a tres seres animados.

qa'tulil yuca "el segundo" < qa'tul "dos" < qa "dos" (ibid.)

Hay semejanzas y diferencias entre las derivaciones que resultan del prefijo del pronombre de la tercera persona combinado con -al o -il. En primer lugar, se aplican en idiomas distintos. Además, en el tsotsil los ordinales derivados funcionan como ordinales sustantivos y adjetivos, y en el chuj llevan un solo aspecto, de ordinales atributos.

\section{III.2. $\mathrm{t}-\ldots$-in / -i'n}

En el análisis de Sywulka, el afijo -in "deriva en el mam los ordinales de los cardinales" (1966: 184). Por sí misma esta unidad no es suficiente para tal tipo de derivación. Después Sywulka menciona que hay que agregar el prefijo del posesivo de la tercera persona (ibid.). En otras palabras, no son los morfemas -in los que derivan los ordinales, sino, como hemos visto en otros idiomas mayances, es la combinación de dos categorías morfémicas: de sintaxis y de derivación. Además, no resultan precisamente los ordinales, "sino en sustantivos concretos de una raíz numérica" (Fran- 
kle, 1980: 152). Por el tipo de derivación, no de los elementos implicados, nos hace recordar el sufijo - $a j$ del quiché, el -al en el tsotsil y el -il en el cakchiquel.

Lo interesante de la unidad morfémica -in, etc., es que tiene el mismo valor morfológico cuando, a partir de otro tipo de raíz nominal "produce en el cakchiquel y en el tseltal de Tenejapa sustantivos denominales concretizados" (ibid.), como los siguientes: tutin tsel T "niño" (Hunn, 1977: 281) < tut tsel "pequeño, chico" (Robles, 1966: 69) ; sots'in cakchi "sitio de murciélagos" < sots' "murciélago" (CE, 1940: 349).

Con referencia al prefijo $t$-, es invariable antes de $\mathrm{C}$ o $\mathrm{V}$, una situación rara en el grupo mayance. El teco es el otro idioma que emplea la $t$ - y el segundo afijo indicado. Para Kaufman el teco es "fonológicamente el más conservador del grupo mameano" (1969: 167). De todos modos hay una diferencia entre el mam y el teco en la aplicación de los morfemas especificados: en el mam deriva un ordinal sustantivo y en el teco un ordinal atributo.

tošin mam "el tercero" < oše "tres" (Sywulka, 1966: 184) / to:șiin teco "tercero" < *o: ș < o:š- proto-mayance "tres" (Kaufman, 1969: 174).

tjo'eb'in teco "quinto" $<$ jo'e' $<$ ho'e' mayance $<$ *ho'- proto-mayance "cinco" (ibid.). twuqin mam "el séptimo" < wuq "siete". tqab"lajin mam "el duodécimo" < qab"laj "doce" (Sywulka, 1966: 184).

\section{III.3. u- . . - ib}

Para formar ordinales de los cardinales del dos en adelante, en el chontal se utiliza el sufijo $-i b$ que no se agrega directamente a la raíz cuantificativa, sino a la raíz numérica que lleva el clasificador específico. Además, "esta combinación de elementos morfémicos requiere $u$ 'su' o $t u$ 'a su' " según el anáilsis de Keller (1955: 273). El primer fonema es el prefijo pronominal del posesivo de la tercera persona ante $\mathrm{C}$, que concretiza el significado de la raíz. En el chontal los ordinales tienen su valor solamente con el clasificador que pertenece al objeto especificado.

učäntekib šan "la cuarta rama de la palma" < u "su"; čän "cuatro"; -tek "el clasificador más común para plantas' (ibid.); šan "palma". 
uyušpets'ib "la tercera vez" < u "su"; yuš "tres" y -pets' "el clasificador para el número de veces" (Op. cit., 263).

Un aspecto que evoca interés con referencia a los idiomas mayances y túrquicos, a pesar de que no presenta nada idéntico, se encuentra precisamente en la formación de los ordinales. En los casos que requieren en el mayance el prefijo que corresponde al pronombre de la tercera persona que sirve también para expresar posesión, en el túquico se utiliza el sufijo del posesivo. La unidad morfémica -nči, etc., del túrquico se analiza "como un posesivo de la tercera persona en $-i$ de una derivación en -nč" (Frankle, 1948: 32). El uygur, uno de los idiomas más antiguos de este grupo, muestra solamente $-n \check{c}$, que no tiene la desinencia $-i$ para formar el posesivo. Dicho de otra manera, es el mayance antiguo que utiliza el prefijo del posesivo con o sin sufijos, según el caso, y en el túrquico es un elemento más reciente en la forma de un sufijo.

birinč uy / birindži / turco / birinči qaračay / birimiči qumyq / birimdži azari "primero" < bir "uno" (ibid.).

onunč uy / onintš̌ tobol / onîndzî baraba küärik / onîndži šor lebed / onunču qyrgyz qrym karaim de Troki / onuntsu karaim de Łuck qoman / onunšu qazaq / onundžu turco / onumču qumyq "décimo" < on "diez" (Op. cit., 83).

La presencia de la / č / en el kar T de la / ts / en el kar $\mathrm{L}$ coincide con la misma alternancia entre el huasteco de Veracruz y el huasteco de San Luis Potosí.

La diferencia en los fonemas consonánticos del sufijo corresponde por completo a la modificación fonológica según el sistema fonémico del idioma. Aquí tenemos una sola unidad morfémica mientras que el mayance emplea una variedad de morfemas y maneras de aplicarlos para expresar el mismo concepto, es decir, los ordinales.

Otra diferencia entre los dos grupos lingüísticos que es muy notable es el hecho de que el grupo túrquico no tiene nada semejante a los clasificadores que caracterizan al mayance en general, con la excepción del huasteco.

Hay un afijo que se utilizó solamente en un idioma túrquico, en el čagatay de Asia Central, que fue notado por Vámberg (1867: 19). Se trata del grupo morfémico -lanč̃ / -lenci que analicé originalmente en -la- / -le- para verbos denominales, .n para sustantivos 
deverbales con el afijo secundario -či (Frankle, 1946:324). Desde el punto de vista de que existe $-n \check{c}$ para los ordinales, más $-\check{i} /-i$, el sufijo del posesivo de la tercera persona, considero ahora -lanč $\check{i}$ una composición de -la-, como anotado, -nč para sustantivos deverbales de muchas categorías, más $-i$, la desinencia para el posesivo. Se ve que el čagatay que pertenece al mismo ramo como el uygur, demuestra la formación más moderna, y además contiene un sufijo adicional que deriva verbos denominales, un aspecto desconocido a los demás idiomas túrquicos en su formación de los ordinales.

birlenči ča "primero" < bir "uno".

ikilenči ča "segundo" < iki "dos".

\section{BIBLIOGRAFIA}

ARZÁPALO, RAMÓN

1973 Das pronominalsystem des Yukatekischen, Fink Verlag - Munchen, Structura - Schriftenreihe zu Lingüistik, Bd. 6.

Aulie, H. WILBUR Y Evelyn W. DE

1978 Diccionario Ch'ol-Español, Español-Ch'ol, Publicado por el Instituto Lingüístico de Verano en coordinación con la Secretaría de Educación Pública a través de la Dirección General de Ser. vicios Educativos en el Medio Indígena, México, D.F.

Barrera Vásquez, Alfredo, Director

1980 Diccionario Maya Cordemex, Maya-Español, Español-Maya, Ediciones Cordemex, Mérida, Yucatán, México.

Ce (véase Sáenz de Santa María)

CORDEMEX (véase BARRERA VÁSQUEZ)

Cowan, Mariat M.

1969 Tzotzil Grammar, Instituto Lingüístico de Verano, A.C., México, D. F.

Delgaty, Alfa

1961 "Notes on Dependent Versus Independent Nouns in Tsotsil", A. William Townsend Cameron en el vigésimoquinto aniversario del Instituto Lingüístico de Verano, editado por B. F. Elson

EDMonson, MUNRo S. y Juan Comas, México, D.F.: 413-420.

1965 Quiche-English Dictionary, Publication 30, Middle American Research Institute, Tulane University, New Orleans.

Frankle, Eleanor

1946 Word Formation in the Turkic Languages, disertación doctoral aceptada; ms., Columbia University, New York.

1948 Word Formation in the Turkic Languages, una parte de la tesis, Columbia University Press, New York.

1978a "Semejanzas entre las derivaciones en las lenguas túrquicas y mayances", ponencia presentada a la ALADAA, México, D.F.

1978b Reduplicación en las lenguas mayances y túrquicas, ms., Depto. de Lingüística, INAH, México. 
1980 Formación de palabras mayances y túrquicas, ms., Depto. de Lingüística, INAH, México.

HaUgen, Einar

1965 "Construction and Reconstruction in Language Planning: Ivar Aasen's Grammar", WORD, Journal of the Linguistic Circle of New York, Vol. 21, number 2, August: 188-207.

Hopkins, Nicholas ARThur

1967 The Chuj Language, Austin, Texas.

Hunn, Eugene S.

1977 Tzeltal Folk Zoology, Academic Press, New York.

Hurley Vda. de Delgaty, Alfa y Agustín Ruiz Sánchez

1978 Diccionario Tzotzil de San Andrés con Variaciones Dialectales, Publicado por el Instituto Lingüístico de Verano en coordinación con la Secretaría de Educación Pública a través de la Dirección General de Educación a Grupos Marginados, México, D.F.

Jagobs, Kenneth and Robert E. Longacre

1967 "Paterns and Rules in Tzotzil Grammar", Foundations of Language, 3: 325:389.

KaUfMan, TERrence

1969 "Teco - a New Mayan Language", IJAL, Vol. XXXV: 154174.

1972 El Proto-Tzeltal-Tzotzil, Fonología Comparada y diccionario reconstruido, CEM, Cuaderno 5, UNAM, México.

Keller, Kathryn C.

1955 "The Chontal (Mayan) Numeral System", IJAL, Vol. XXI, No. $3: 258-275$.

LaFarge, Oliver $I I$ and Douglas Byers

1931 The Year-Bearer's People, The Department of Middle American Research, The Tulane University of Louisiana, New Orleans, La.

LARSEN, RAMÓN

1955 Vocabulario Huasteco del Estado de San Luis Potosí, Publicado por el Instituto Lingüístico de Verano y SEP, México, D.F.

Lázaro Garreter, Fernando

1971 Diccionario de Términos Filológicos, Editorial Gredos, S.A., Madrid.

Mayers, Marvin K., Editor

1966 Languages of Guatemala, Janua Linguarum, Series Práctica 23, Mouton Co., London - the Hague - Paris.

Michelon, Oscar, Ed.

1976 Diccionario de San Francisco, Akademischer Druck und Verlagsanstalt, Graz, Austria.

Ochoa Peralta, Ma. Ángela

1978 Tesis, El idioma Huasteco de Xil-Xuchil, Veracruz, INAH.

Robles Uribe, Carlos

1966 La Dialectología Tzeltal y el Diccionario Compacto, México, INAH, Publicaciones 6 .

Sáenz de Santa María, Carmelo

1940 Diccionario Cakchiquel-Español, Guatemala, CA. 
Schumann, Otto

1973 La Lengua Chol de Tila (Chiapas), CEM, UNAM, México, Cuaderno 8.

1976-77 "Notas sobre numerales y el plural en el Quiché de Sajcabaja", Estudios de Cultura Maya, Vol. X:243-252 CEM, UNAM, México.

SF (véase Michelon)

Swadesh, Mauricio

1966 "Porhé y Maya", Anales de Antropología, UNAM, Vol. 3: 173-204.

Swadesh, Mauricio, Ma, Cristina Álvarez y Juan G. Bastarrácheá

1970 Diccionario de Elementos del Maya Yucateco Colonial, Seminario de Estudios de la Escritura Maya, Cuaderno 3, UNAM, México.

SYWULKa, EDWARD

1966 "Mam Grammar", Languages of Guatemala, 178-195.

VAMBERY, H.

1867 Cagataische Sprachstudien, Leipzig.

Warkentin, Viola M. y Ruth M. Brend

1974 "Chol Phonology", Linguistics, 132, July: 87-102. 